\title{
Ultrasound-guided erector spinae plane (ESP) block: A novel intervention for mechanical back pain in the emergency department
}

\author{
Yajai Apibunyopas, MD*; Ben Ho, BSc, MD*
}

Keywords: back pain, emergency department, erector spinae plane block, ultrasound-guided regional anesthesia

\section{INTRODUCTION}

Lower back pain (LBP) is one of the most common health problems worldwide ${ }^{1-3}$ and is the leading cause of work absenteeism. ${ }^{4}$ LBP accounts for $3 \%-4 \%$ of all emergency department (ED) presentations, ${ }^{5}$ and current ED analgesic options include acetaminophen, nonsteroidal anti-inflammatory drugs (NSAIDs), and opioids. ${ }^{6,7}$ With the current worldwide opioid epidemic and significant opioid-related adverse effects, having an additional opioid-sparing therapeutic option in the ED would be desirable. Ultrasound-guided erector spinae plane (ESP) block is a novel regional anesthesia technique recently described for thoracic regional pain control and analgesia. ${ }^{7-12}$ It has been described as an analgesic technique for treating neuropathic thoracic pain, pain associated with rib fractures, and postthoracotomy pain. Previously unreported therapeutic use of the ESP block for mechanical back pain is described here using 12 blocks in 10 ED patients.

\section{POPULATION}

All cases were adult patients (age $\geq 18$ years old) who presented to the ED with mechanical back pain of moderate to severe intensity for whom an ultrasound-guided ESP block was one of the methods of pain management during May to June 2017. The authors were consulted by the primary emergency physician to perform the ESP block for pain control during scheduled "procedure shifts" for the hospital. Patients received ancillary treatment, including non-opiate pre-block analgesics as dictated by the primary emergency physician. No patients had historical red flags (fever, symptoms of cauda equina, cancer history, progressive neurologic symptoms), evidence of skin/soft tissue infection at the block site, or suspected coagulopathy. All patients provided written informed consent before the procedure. The ESP block was performed in the same manner in each case by one of the two authors. Patients' verbal pain numeric rating scale (NRS) from 0-10 were recorded pre-block and 15 minutes to 1 hour post-block. The presence of immediate complications of bleeding or new neurologic symptoms were also recorded at the time of post-block NRS recording. Acetaminophen and ibuprofen (if no contraindications) were suggested for ongoing analgesia post-ED discharge. A post-discharge telephone follow-up to determine block duration and presence of any patient reported delayed complications was performed between 2 and 30 days post-block by one of the two authors.

\section{BLOCK TECHNIQUE}

The goal of the ESP block is to place a local anesthetic between the erector spinae muscle (ESM) and the underlying vertebral transverse process. The ESP block is thought to block the common dorsal rami,

From the *Emergency Department, Nanaimo Regional General Hospital, Nanaimo, BC.

Correspondence to: Dr. Yajai Apibunyopas, Nanaimo Regional General Hospital, 1200 Dufferin Crescent, Nanaimo, BC V9S 2B7;

Email: jo2jo25@gmail.com

(c) Canadian Association of Emergency Physicians

CJEM 2019;21(2):302-305

DOI 10.1017/cem.2018.469 
medial branches, and lateral branches of spinal nerves and also their anastomoses.

The patient is positioned in a lateral decubitus position with the side of maximum tenderness up. Prone positioning, if tolerated by the patient, may also be used. The ultrasound transducer is placed in the longitudinal orientation over the spinous process of the vertebra corresponding to the level of maximum tenderness. The probe is then slid laterally to center the lateral tip of the target transverse process on the screen. The ESM identified near-field to the transverse process. Skin anesthesia is achieved with $1 \%$ lidocaine, then an $8 \mathrm{~cm}, 22$-gauge spinal needle is inserted in-plane under ultrasound guidance until the needle tip is visualized in the interfascial plane between the ESM and the transverse process. An intermittent bolus injection of $1 \%$ lidocaine while advancing the needle helps "hydro-localize" and track the needle tip down to the target plane (Figures 1 and 2). When the needle tip is within the plane far field to the ESM as confirmed by a visualization of a hypoechoic longitudinal spread of the local anesthetic, the anesthetic agent is switched to $20 \mathrm{ml}$ of $0.25 \%$ bupivacaine without epinephrine to complete the block. The use of pediatric extension tubing, a three-way stopcock, and an assistant to push the anesthetic facilitate the performance of the block. An aspiration attempt prior to the injection of bupivacaine is performed to detect an inadvertent dural or intravascular puncture.

Although not specifically used in these cases, bilateral ESP blocks may be used for those with significant

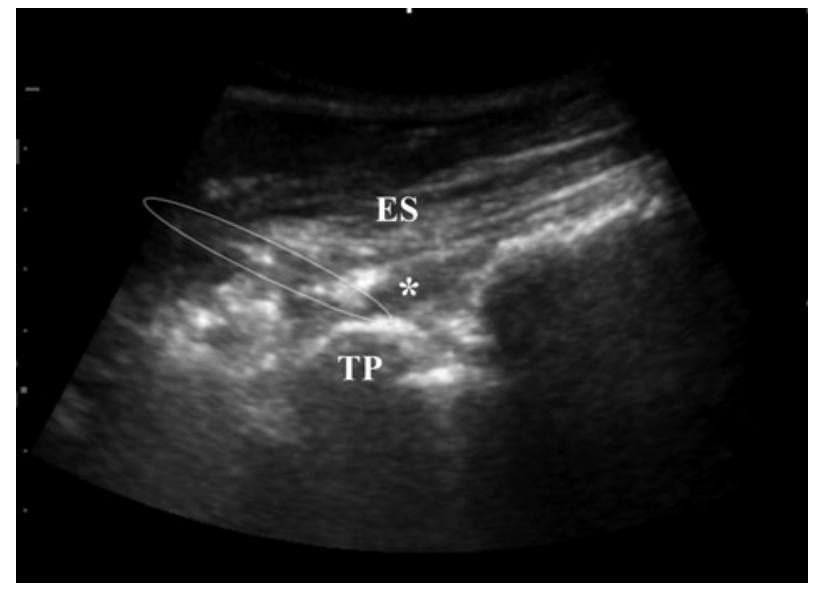

Figure 1. The ultrasound image shows the needle (oval) visualized with the tip just nearfield to the transverse process (TP) and farfield to the overlying erector spinae muscle (ES). Hypoechoic spread of local anaesthetic $\left({ }^{*}\right)$ is seen tracking along the fascial plane deep to the muscle.

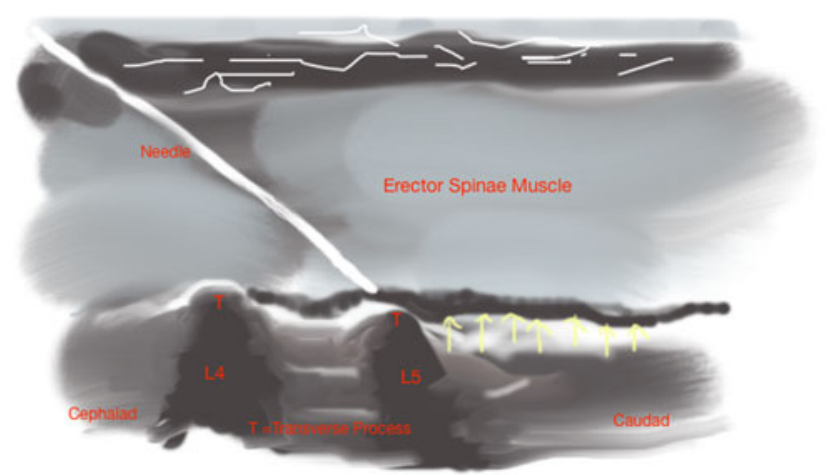

Figure 2. Illustration shows injection of anesthetic agent into the interfascial plane deep to ESM producing a hypoechoic linear pattern of local anesthetic spread (arrows).

tenderness/spasm bilaterally. As always, local anesthetic toxicity dosage limits must be considered.

\section{RESULTS}

Twelve ESP blocks were used therapeutically in 10 ED patients for mechanical back pain; nine presented with LBP and one with thoracic back pain. There were nine block responders and one non-responder. One patient had three ESP blocks over a 7-week period for her chronic LBP. A summary of the cases is included in Appendix 1.

In our experience, a significant clinical effect was observed between 15 minutes and 1-hour post-block. The analgesic duration ranged from 36 hours up to 4 weeks in responders. Mean block duration was 8.2 days. Mean initial NRS score reduction was 5.9 in responders, 0 in the non-responder, and 5.4 overall.

Two patients presented were unable to mobilize from the ED stretcher due to pain but were subsequently discharged ambulatory, possibly preventing a prolonged ED stay or hospital admission. The only complication reported in this series was local tenderness at the site of injection.

\section{DISCUSSION}

We first discovered the significant paravertebral pain relief when performing an ultrasound-guided ESP block on a patient with a rib fracture and concomitant paravertebral pain. The paravertebral pain was completely resolved (while the rib pain was partially resolved), which led to the hypothesis that this block 
could provide benefit to patients with back pain, either mechanical or neuropathic.

In 2016, Forero first introduced the ultrasoundguided ESP block for treatment of thoracic pain in patients with metastatic disease involving ribs and patients with a malunion of multiple rib fractures. Subsequently, he reported its effectiveness in patients with chronic thoracic neuropathic pain and acute surgical pain from thoracotomy. ${ }^{10}$ Another study later applied this technique for regional anesthesia for abdominal surgery by performing the block at the lower thoracic level. ${ }^{13}$

Although recent evidence-based guidelines on back pain treatment offer other available interventions such as targeted at facet joint, medial branch, and sacroiliac joint injections, these procedures require special equipment and training not typically available in the ED setting. ${ }^{6,14-16}$ The ultrasound-guided ESP block is a promising therapeutic intervention for mechanical back pain treatment in the ED. Both the equipment and the skill set required are within the scope of the practising emergency physician.

We agree with previous authors ${ }^{7,10,13}$ who postulated that the ESP block provides pain relief by blocking the common dorsal rami, medial branches, and lateral branches of spinal nerves as well as their anastomoses. ${ }^{17-19}$ The ESM extends over the length of the thoracolumbar spine, and the potential space deep to the muscle allows extensive cranio-caudal local anesthetic spread along this plane, allowing multiple levels to be affected with a single injection.

The average NRS reduction noted in our series is well above the threshold for minimal clinically meaningful pain relief reported in the literature across varied patient populations of $1.3-2.4,{ }^{20-23}$ including a study looking at an ED-specific population where the threshold was $1.3 .^{20}$ The NRS reductions seen in our series (5.9 in responders and 5.4 overall) compare favorably with the reported mean pain score reductions of $4.1-4.5$, using $0.1 \mathrm{mg} / \mathrm{kg}$ morphine intravenously for treatment of acute painful conditions in the $\mathrm{ED}$ ( $\mathrm{n}=478$ in two studies). ${ }^{24,25}$

\section{CONCLUSION}

We describe here a novel treatment for mechanical back pain in the ED setting using the ultrasound-guided ESP block in a case series of 10 patients. There was a significant pain reduction with the block. Adverse effects of prescription opioids use are a major current worldwide healthcare crisis, and the ESP block has the potential to offer an opioid-sparing analgesic option for the ED treatment of mechanical back pain. This analgesic technique warrants further research, comparing the efficacy of the ESP block with standard analgesic ED treatment for mechanical back pain.

\section{SUPPLEMENTARY MATERIAL}

The supplementary material for this article can be found at https://doi.org/10.1017/cem.2018.469.

Competing interests: None declared.

\section{REFERENCES}

1. Andersson GB. Epidemiology of low back pain. Acta Orthop Scand Suppl 1998;281:28-31.

2. Dionne CE, Dunn KM, Croft PR. Does back pain prevalence really decrease with increasing age? A systematic review. Age Ageing 2006;35(3):229-34.

3. Deyo RA, Cherkin D, Conrad D, Volinn E. Cost, controversy, crisis: low back pain and the health of the public. Ann Rev Public Health 1991;12:141-56.

4. Lidgren L. The bone and joint decade 2000-2010. Bull World Health Organ 2003;81(9):629.

5. Friedman BW, Chilstrom M, Bijur PE, Gallagher EJ. Diagnostic testing and treatment of low back pain in United States emergency departments: a national perspective. Spine 2010;35(24):E1406-11.

6. Manchikanti L, Abdi S, Atluri S, et al. An update of comprehensive evidence-based guidelines for interventional techniques in chronic spinal pain. Part II: guidance and recommendations. Pain Physician 2013;16(2 Suppl):S49-283.

7. Forero M, Adhikary SD, Lopez H, et al. The erector spinae plane block: a novel analgesic technique in thoracic neuropathic pain. Reg Anesth Pain Med 2016;41(5):621-7.

8. Ueshima H, Otake H. Clinical experiences of ultrasoundguided erector spinae plane block for thoracic vertebra surgery. 7 Clin Anesth 2017;38:137.

9. Hamilton DL, Manickam B. Erector spinae plane block for pain relief in rib fractures. Brit $\mathcal{F}$ Anaesth 2017;118 (3):474-5.

10. Forero M, Rajarathinam M, Adhikary S, Chin KJ. Continuous erector spinae plane block for rescue analgesia in thoracotomy after epidural failure: a case report. A \& A case reports; 2017.

11. El-Boghdadly K, Pawa A. The erector spinae plane block: plane and simple. Anaesthesia 2017;72(4):434-8.

12. Hamilton DL, Manickam B. The erector spinae plane block. Reg Anesth Pain Med 2017;42(2):276.

13. Chin KJ, Adhikary S, Sarwani N, Forero M. The analgesic efficacy of pre-operative bilateral erector spinae plane (ESP) 
blocks in patients having ventral hernia repair. Anaesthesia 2017;72(4):452-60.

14. Peh WCG. Image-guided facet joint injection. Biomed Imaging Interv 7 2011;7(1):e4.

15. Zhou L, Schneck CD, Shao Z. The anatomy of dorsal ramus nerves and its implications in lower back pain. Neurosci Med 2012;3(2):10.

16. Rados I, Elezovic N. Lumbar facet joint injection and medial branch block. Period Biol 2013;115(2):155-8.

17. Shuang F, Hou S-X, Zhu J-L, et al. Clinical anatomy and measurement of the medial branch of the spinal dorsal ramus. Medicine 2015;94(52):e2367.

18 . Bogduk N, Long DM. Percutaneous lumbar medial branch neurotomy: a modification of facet denervation. Spine 1980;5 (2):193-200.

19. Miyakoshi N, Shimada Y, Kasukawa Y, et al. Total dorsal ramus block for the treatment of chronic low back pain: a preliminary study. Foint Bone Spine 2007;74(3):270-4.

20. Bijur PE, Latimer CT, Gallagher EJ. Validation of a verbally administered numerical rating scale of acute pain for use in the emergency department. Acad Emerg Med 2003;10(4):390-2.
21. Kendrick DB, Strout TD. The minimum clinically significant difference in patient-assigned numeric scores for pain. Am 7 Emerg Med 2005;23(7):828-32.

22. Sloman R, Wruble AW, Rosen G, Rom M. Determination of clinically meaningful levels of pain reduction in patients experiencing acute postoperative pain. Pain Manag Nurs 2006;7(4):153-8.

23. Hjermstad MJ, Fayers PM, Haugen DF, et al. Studies comparing Numerical Rating Scales, Verbal Rating Scales, and Visual Analogue Scales for assessment of pain intensity in adults: a systematic literature review. 7 Pain Symptom Manage 2011;41(6):1073-93.

24. Birnbaum A, Esses D, Bijur PE, et al. Randomized double-blind placebo-controlled trial of two intravenous morphine dosages $(0.10 \mathrm{mg} / \mathrm{kg}$ and $0.15 \mathrm{mg} / \mathrm{kg})$ in emergency department patients with moderate to severe acute pain. Ann Emerg Med 2007;49(4):445-53, 53. e1-2.

25. Chang AK, Bijur PE, Meyer RH, et al. Safety and efficacy of hydromorphone as an analgesic alternative to morphine in acute pain: a randomized clinical trial. Ann Emerg Med 2006;48(2):164-72. 